\title{
Analyse de sensibilité des flux de chaleur pariétaux en canal asymétriquement chauffé au moyen d'une corrélation permettant d'estimer les transferts de chaleur
}

\section{Analysis of wall heat flux sensibility in asymmetrically heated channel with a heat transfer correlation}

\author{
Martin David $^{1 *}$, Adrien Toutant ${ }^{1}$, Françoise Bataille $^{1}$ \\ ${ }^{1}$ Laboratoire PROMES-CNRS (UPR 8521), Université de Perpignan via Domitia, Tecnosud-Rambla de la \\ thermodynamique, 66100 Perpignan - France, " martin.david@ promes.cnrs.fr
}

RÉSUMÉ. Les travaux concernent l'étude d'une corrélation permettant d'estimer les transferts de chaleur dans des conditions de forts chauffages asymétriques d'écoulements turbulents en canal caractérisés par de hauts niveaux de températures. La corrélation vise à faciliter l'estimation des transferts de chaleur dans les récepteurs solaires à gaz souspression des centrales solaires à tour. Une étude de sensibilité des flux de chaleur pariétaux aux différents paramètres de l'écoulement est menée. La propagation des incertitudes de mesures sur les résultats est quantifiée en fonction de la position axiale dans le récepteur solaire puis en fonction du rapport des températures de fluide et de paroi froide.

SUMMARY. This paper study a heat transfer correlation for asymetrically heated turbulent channel flows characterized by high temperature levels. The correlation aims to estimate heat transfers in gas pressurized solar receiver of concentrated solar power tower. A wall heat fluxes sensitivity study to flow parameters is performed. The propagation of measurement uncertainties is quantified as a function of the axial location in the solar receiver and, then, as a function of the fluid-to-wall temperature ratio.

MOTS-CLÉS. Transferts de chaleur, Corrélation, Propagation des incertitudes, Récepteurs solaires, Écoulements turbulents.

KEYWORDS. Heat transfers, Correlation, Uncertainty propagation, Solar Receiver, Turbulent flows.

\section{Nomenclature}

\begin{tabular}{|l|l|l|l|}
\hline Symbole & Signification, unités & Symbole & Signification, unités \\
\hline$D_{h}$ & Diamètre hydraulique, $m$ & \multicolumn{2}{|c|}{ Indices et exposants } \\
\hline$N u$ & nombre de Nusselt & $b$ & moyenne \\
\hline$R e$ & nombre de Reynolds & $p$ & paroi \\
\hline$P r$ & nombre de Prandtl & $\bar{p}$ & paroi opposée \\
\hline$T$ & température, $\mathrm{K}$ & $c h$ & chaud \\
\hline \multicolumn{2}{|c|}{ Symboles grecs } & $f r$ & froid \\
\hline$\lambda$ & conductivité thermique, $\mathrm{W} \mathrm{m}^{-1} \mathrm{~K}^{-1}$ & & \\
\hline$\Phi$ & flux de chaleur, $\mathrm{W} \mathrm{m} \mathrm{m}^{-2}$ & & \\
\hline
\end{tabular}




\section{Introduction}

La détermination des transferts thermiques dans des systèmes complexes requiert des moyens expérimentaux ou numériques conséquents. Les corrélations permettant de calculer le nombre de Nusselt permettent d'estimer les transferts thermiques, généralement, avec une erreur inférieure à $20 \%$ [1, 2, 3, 4, 5]. Elles sont donc des outils simples d'utilisation et particulièrement bien adaptées à l'ingénierie pour le pré-dimensionnement de systèmes tels que des échangeurs de chaleur, des systèmes électroniques ou des récepteurs solaires.

Les récepteurs solaires à gaz sous-pression des centrales solaires à tour sont le lieu d'écoulements fortement turbulents chauffés asymétriquement à hauts niveaux de températures [6]. Les conditions extrêmes des récepteurs solaires complexifient les mesures des paramètres de l'écoulement. Ainsi, les incertitudes sur les mesures peuvent être la cause de mauvaises estimations des flux de chaleur pariétaux. Il est donc important de quantifier la propagation des erreurs liée à ces incertitudes. En étudiant les transferts convectifs en jeu dans l'industrie alimentaire, Hakansson [7] a noté que le coefficient de transfert thermique varie de plusieurs ordres de grandeur selon les études. Ses travaux, s'appuyant sur la méthode GUM (Guide pour l'expression de l'incertitude de mesure) [8], ont montré que certaines méthodes d'estimation du coefficient de transfert thermique sont très dépendantes des incertitudes de mesure. Les écarts trouvés dans la littérature sont donc probablement liés à ces dernières.

Les corrélations peuvent servir de base afin de quantifier la sensibilité des tranferts thermiques aux différents paramètres de l'écoulement. Par exemple, Driscoll et Landrum [9] ont utilisé une corrélation afin de quantifier la propagation des incertitudes lors de la détermination des transferts de chaleur au sein de moteurs employés dans l'aérospatiale. Ils ont ainsi pu déterminer l'impact des incertitudes dans leur conception. Scariot et al. [10] ont analysé la sensibilité de la température du fluide et de l'enthalpie, obtenues par un calcul impliquant des corrélations de transfert de chaleur, aux incertitudes de mesure. Ils étudient un écoulement de $\mathrm{CO}_{2}$ dans un tube, entrant à l'état gazeux et sortant à l'état supercritique. Les résultats montrent que l'estimation de la température de fluide est moins sensible aux incertitudes lorsque les conditions d'études sont proches du point critique. Pour l'enthalpie, plus on s'approche du point critique, plus les incertitudes sont élevées.

La présente étude vise à quantifier la sensibilité des flux de chaleur pariétaux, lors de la détermination des transferts de chaleur dans les récepteurs solaires à gaz sous-pression des centrales solaires à tour, au moyen d'une corrélation. La corrélation de David et al. [11], développée afin d'estimer les transferts thermiques dans les conditions de fonctionnement des récepteurs solaires, est retenue pour mener cette étude.

Dans un premier temps, la corrélation proposée est présentée dans la section 2. Dans un second temps, une étude de sensibilité du flux de chaleur aux différents paramètres de l'écoulement est menée grâce à cette corrélation dans la section 3.

\section{Corrélation pour les transferts thermiques fluide-paroi en condition de chauffage asymé- trique}

La corrélation proposée par David et al. [11] est destinée à l'estimation des transferts thermiques dans les conditions de fonctionnement des récepteurs solaires à gaz sous-pression. Elle a été établie grâce à 70 Simulations des Grandes Échelles de turbulence (SGE), permettant d'obtenir un échantillon de 


\begin{tabular}{ll}
\hline Chauffage symétrique & Chauffage asymétrique \\
\hline $12000<R e_{b}<177000$ & $10600<R e_{b}<145000$ \\
$0.47<T_{b} / T_{p}<0.99$ & $1.1<T_{p c h} / T_{p f r}<2.0$ \\
& $0.63<T_{b} / T_{p f r}<0.95$ \\
& $0.44<T_{b} / T_{p c h}<0.85$ \\
\hline
\end{tabular}

TABleau 1. Plage de nombre de Reynolds et ratios de températures couverts par la corrélation.

données fiable et conséquent. Durant les simulations, les équations de Navier-Stokes sont résolues sous l'hypothèse de bas nombre de Mach dans un canal plan bi-périodique. Les couplages entre la thermique et la dynamique de l'écoulement (en particulier les effets de dilatation liés à la température) sont pris en compte afin de se rapprocher des conditions réelles de fonctionnement des récepteurs solaires. La gravité, dont l'influence est négligeable dans les configurations étudiées, n'intervient pas dans les équations. Les petites échelles de turbulence, non résolues en SGE, sont modélisées à l'aide du modèle sousmaille fonctionnel Anisotropic-Minimum-Dissipation (AMD) [12]. La corrélation proposée est décrite par l'équation 1 :

$$
N u_{p}=0.024 \operatorname{Re}_{b}^{0.8} \operatorname{Pr}_{b}^{0.4}\left(\frac{T_{p}}{T_{b}}\right)^{-0.9}\left(\frac{T_{p}}{\left|T_{p}-T_{b}\right|}\right)^{1.4\left(1-\frac{T_{p}}{T_{m}}\right) \frac{T_{b}}{T_{p}}}
$$

où $\mathrm{T}_{\mathrm{m}}$ est la moyenne des températures de paroi, le nombre de Reynolds est calculé à partir du diamètre hydraulique du canal. La corrélation est en cohérence avec des expressions de références existantes [1, $2,3,4]$ et fait intervenir un nouveau terme, dédié au chauffage asymétrique. Ce terme devient neutre en cas de chauffage symétrique du fluide rendant la corrélation également utilisable dans ces conditions.

\subsection{Domaine d'application de la corrélation}

Cette corrélation couvre un large domaine d'application : les températures de paroi varient de $293 \mathrm{~K}$ à $1300 \mathrm{~K}$, la température de fluide couvre la gamme $342 \mathrm{~K}-1237 \mathrm{~K}$, le nombre de Prandtl est compris entre 0,76 et 3,18 et les flux de chaleur varient entre $4 \mathrm{~kW} / \mathrm{m}^{2}$ et $578 \mathrm{~kW} / \mathrm{m}^{2}$. Le tableau 1 précise la gamme de nombre de Reynolds ainsi que les ratios de températures pour lesquels la corrélation est valide.

\subsection{Validation de la corrélation}

Les nombres de Nusselt obtenus avec la corrélation proposée par David et al. [11] sont tracés en fonction des nombres de Nusselt obtenus par les simulations numériques sur la figure 1. La gamme de Nusselt étudiée varie de 20 à 680. Les résultats montrent que l'ensemble des points est contenu dans la marge d'erreur de $10 \%$.

\section{Analyse de sensibilité de l'estimation des flux de chaleur pariétaux aux paramètres de l'écoulement}

La sensibilité de l'estimation des flux de chaleur pariétaux aux paramètres de l'écoulement est étudiée dans cette section. Les conditions de cette étude sont caractéristiques des conditions de fonctionnement 


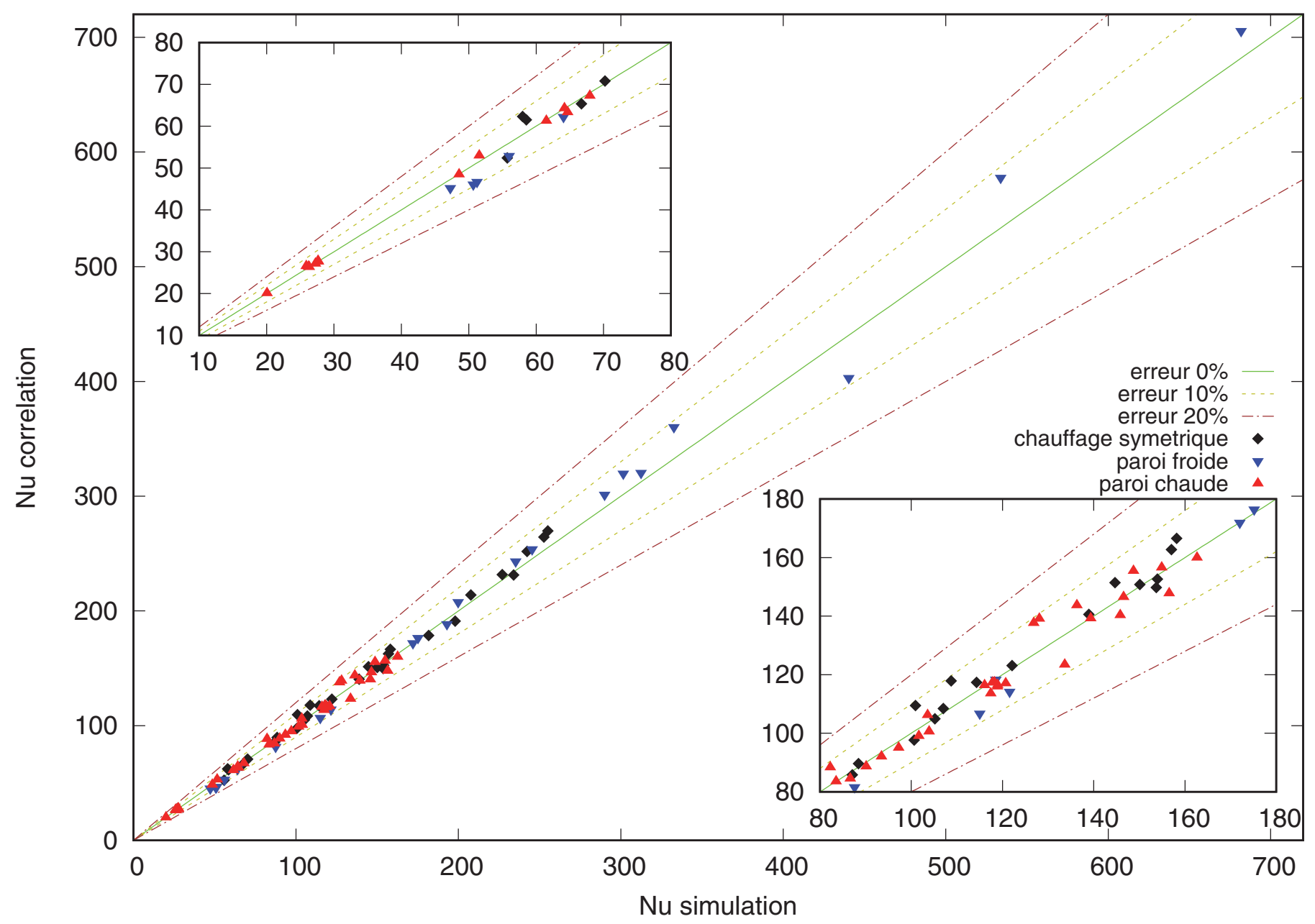

Figure 1. Comparaison du nombre de Nusselt obtenu par la corrélation avec le nombre de Nusselt obtenu par la simulation.

\begin{tabular}{ccccc}
\hline $\operatorname{Re}_{\mathrm{b}}$ & $\operatorname{Pr}$ & $\mathrm{T}_{\mathrm{pch}}[\mathrm{K}]$ & $\mathrm{T}_{\mathrm{pfr}}[\mathrm{K}]$ & $\mathrm{T}_{\mathrm{b}}[\mathrm{K}]$ \\
60000 & 0,87 & 1300 & 900 & 700 \\
\hline
\end{tabular}

TABleau 2. Conditions de l'étude de sensibilité des flux pariétaux aux incertitudes de mesures des paramètres de l'écoulement.

des récepteurs solaires à gaz sous-pression des centrales solaires à tour (voir tableau 2). Les flux de chaleur pariétaux sont calculés à partir des nombres de Nusselt obtenus avec la corrélation à l'aide de l'équation 2. Ils sont de $140 \mathrm{~kW} / \mathrm{m}^{2}$ à la paroi froide et de $250 \mathrm{~kW} / \mathrm{m}^{2}$ à la paroi chaude.

$$
\phi_{p}=\frac{\lambda_{p}\left(T_{p}-T_{b}\right) N u_{p}}{D_{h}}
$$

Dans la suite de l'article, les résultats sur les incertitudes de flux sont obtenus en calculant directement le nombre de Nusselt à partir de la corrélation proposée par David et al. [11] pour différentes valeurs des paramètres de l'écoulement. 


\subsection{Influence des incertitudes de mesures sur l'estimation des flux pariétaux}

Les incertitudes sur les flux pariétaux sont déterminées pour les conditions d'étude de référence décrites dans cette section. Le ratio de température fluide-paroi froide est alors de 0,78. Les incertitudes sur les flux pariétaux sont exposées, sur la figure 2, en fonction de l'incertitude sur les températures de paroi chaude et froide, sur la température de fluide, sur le nombre de Reynolds et sur le nombre de Prandtl. Comme attendu, les résultats montrent que les imprécisions de mesure sur la température de paroi froide (respectivement chaude) sont celles qui ont le plus d'impact sur l'estimation du flux de la paroi froide (respectivement chaude). L'incertitude sur la mesure de la température de fluide est également un facteur important pour la prédiction du flux de la paroi froide. La paroi chaude est, quant à elle, moins impactée. Ces différences sont dues à l'asymétrie du chauffage du fluide : la température de paroi froide est plus proche de la température de fluide que la paroi chaude. Ainsi, une petite variation de la température de fluide aura plus d'influence sur le résultat du flux obtenu à la paroi froide qu'à la paroi chaude. Les températures des parois opposées aux flux concernés ont aussi une influence sur le résultat, même si elle est limitée. La propagation des erreurs liées aux incertitudes sur les nombres de Reynolds et Prandtl est elle aussi moindre. En effet, pour les quatre derniers paramètres abordés, les incertitudes sur les flux sont atténuées. Par exemple, une sous-estimation de la température de paroi chaude de $6 \%$ conduit à une sous-estimation du flux de la paroi froide de 5\%. Enfin, il est intéressant de noter que la propagation des incertitudes concernant les températures n'est pas symétrique : la surestimation de la température de fluide conduit à une erreur moins importante sur le flux de la paroi froide que la sous-estimation de la température de fluide (pour une incertitude de $10 \%$ sur la température on obtient une incertitude sur le flux de $20 \%$ pour la surestimation contre $13 \%$ dans le cas de la sous-estimation).

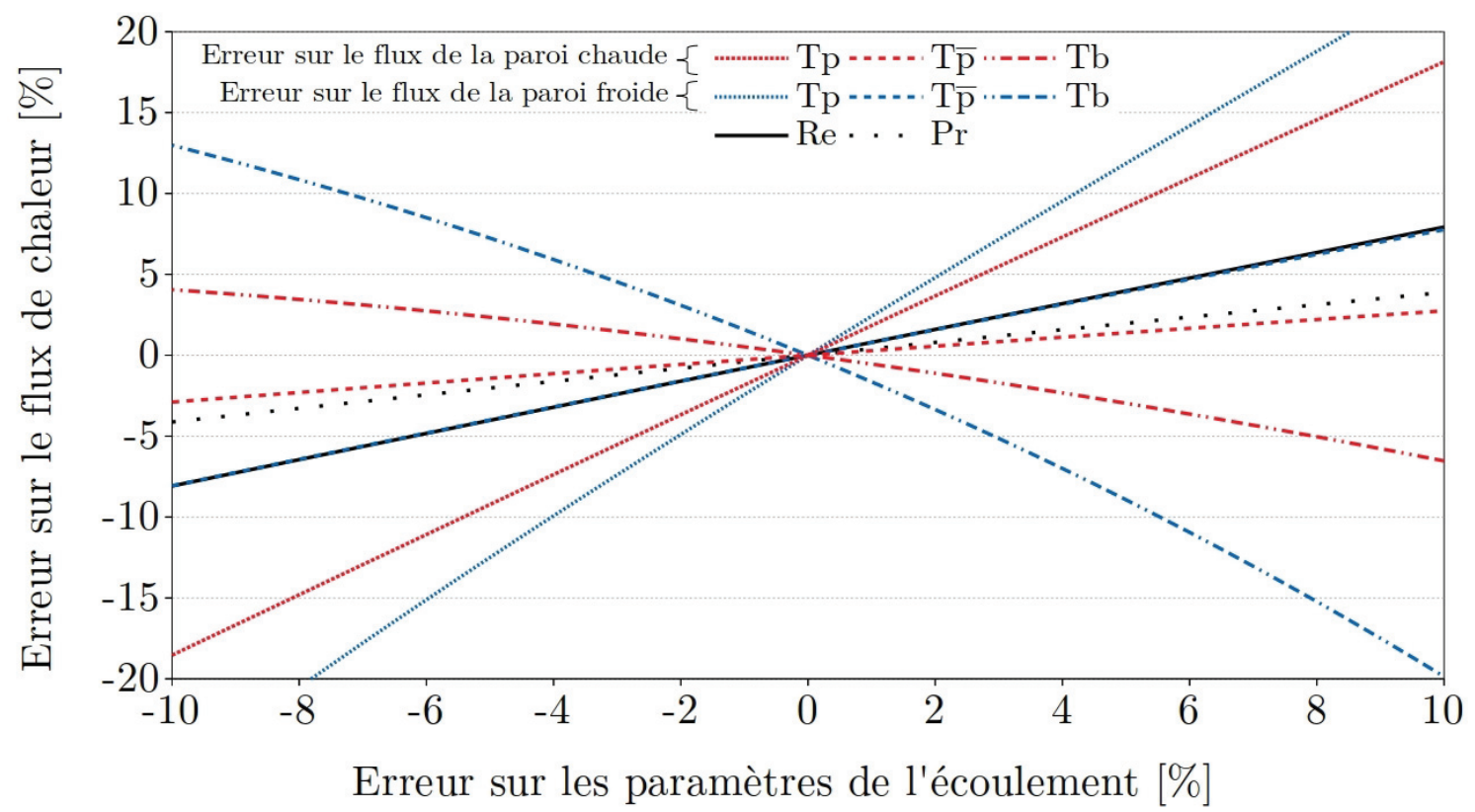

Figure 2. Erreur commise sur le flux pariétal en fonction de l'incertitude des mesures des paramètres influents sur le transfert de chaleur.

Dans les récepteurs solaires à gaz sous-pression, les températures de l'air et celle des parois évoluent en fonction de la position axiale dans le récepteur solaire. Ainsi, la sensibilité des flux de chaleurs pour différents niveaux de température est étudiée. Le tableau 3 résume les configurations étudiées sur la figure 3. La température de paroi chaude varie entre $700 \mathrm{~K}$ et $1300 \mathrm{~K}$. L'écart de température entre les 
deux parois est de $200 \mathrm{~K}$ dans tous les cas considérés et la température moyenne de fluide est inférieure à la température de paroi froide de $150 \mathrm{~K}$. Ces configurations d'étude sont représentatives des conditions opératoires des récepteurs solaires [13].

La figure 3 représente la pente de l'erreur commise sur le flux pariétal en fonction des paramètres de l'écoulement et des températures de paroi. Les pentes sont obtenues en approchant les courbes observées sur la figure 2 par des droites et en déterminant le coefficient directeur des droites dans les différentes configurations listées dans le tableau 3. Le nombre de Reynolds est fixé à 60000 . Les résultats montrent que la pente de la propagation d'erreur augmente avec le ratio de température $T_{b} / T_{p f r}$. La propagation des erreurs sur le flux de la paroi froide est plus sensible aux conditions d'étude que celle commise à la paroi chaude pour les trois grandeurs observées $\left(T_{p}, T_{\bar{p}}\right.$ et $\left.T_{b}\right)$. L'écart entre les erreurs commises sur les flux chaud et froid s'accentue à mesure que le ratio $T_{b} / T_{p f r}$ augmente. Par exemple, pour les conditions $T_{b} / T_{p f r}=0.7$ la pente de l'erreur est de 1,9 à la paroi chaude contre 2 à la paroi froide alors que lorsque le ratio $T_{b} / T_{p f r}$ est égal à 0,86 , la pente de l'erreur est de 3 à la paroi chaude contre 5 à la paroi froide. Il apparaît aussi que l'influence de l'erreur sur la température de fluide augmente plus fortement que celle de la température de paroi.

TABleau 3. Liste des configurations étudiées sur la figure 3.

\begin{tabular}{cccc}
\hline $\mathrm{T}_{\mathrm{pch}}[\mathrm{K}]$ & $\mathrm{T}_{\mathrm{pfr}}[\mathrm{K}]$ & $\mathrm{T}_{\mathrm{b}}[\mathrm{K}]$ & $\mathrm{T}_{\mathrm{b}} / \mathrm{T}_{\mathrm{pfr}}$ \\
\hline 700 & 500 & 350 & 0,70 \\
900 & 700 & 550 & 0,79 \\
1100 & 900 & 750 & 0,83 \\
1300 & 1100 & 950 & 0,86 \\
\hline
\end{tabular}
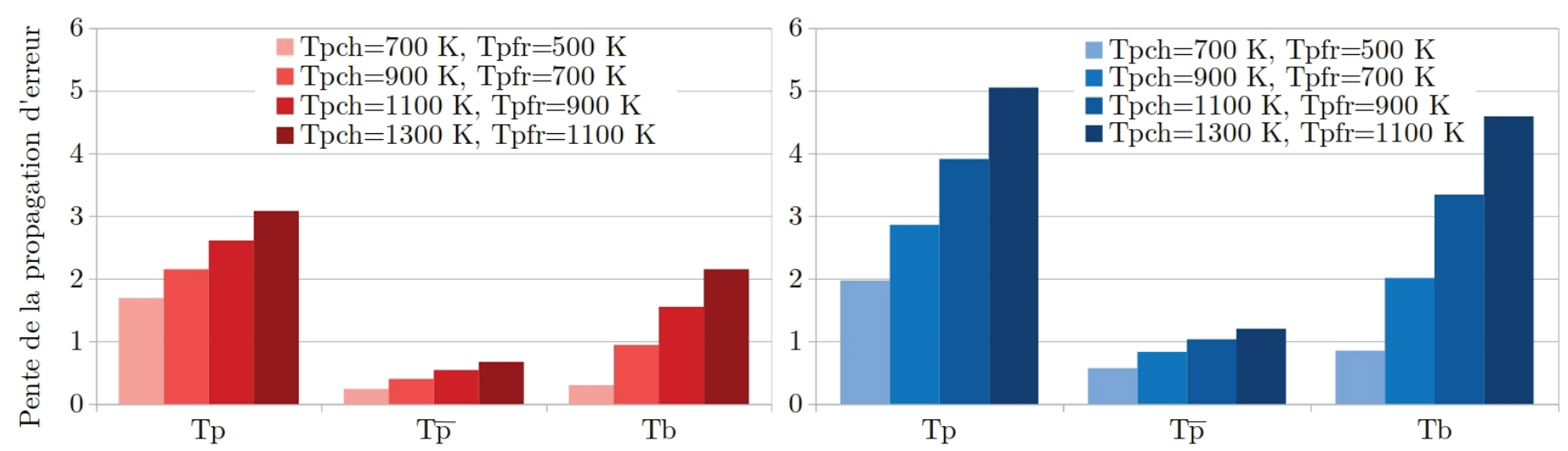

Figure 3. Pente de l'erreur commise sur le flux pariétal en fonction des paramètres de l'écoulement et des températures de paroi. Le nombre de Reynolds est fixé à 60000

La présente section a mis en évidence les paramètres de l'écoulement les plus influents sur les transferts de chaleur. Elle a aussi permis d'étudier l'erreur sur les flux en fonction des niveaux de température. La section suivante aborde l'évolution de l'incertitude sur le flux pour différents ratios de température fluideparoi. 


\subsection{Effet du ratio de température fluide-paroi sur la propagation des incertitudes}

Les incertitudes sur le flux sont dépendantes des incertitudes de mesures mais aussi des conditions de l'écoulement. Dans ce qui suit, les conditions de références de l'étude, détaillées dans la section 3, sont conservées sauf la température du fluide qui varie de façon à couvrir la gamme de validité de la corrélation. Les résultats sont donc présentés pour la plage $0,63<\mathrm{T}_{\mathrm{b}} / \mathrm{T}_{\mathrm{pfr}}<0,95$. Pour un ratio de température de 0.63 , la température de fluide est de $567 \mathrm{~K}$, ce qui correspond à la température d'entrée du fluide dans le récepteur solaire. Les courbes sont tracées pour cinq incertitudes sur les mesures de température : $0,5 \%, 1 \%, 2 \%, 4 \%$ et $8 \%$. L'évolution des flux pariétaux est observée sur l'axe des ordonnées secondaire. Les résultats sont adimensionnés comme explicité dans l'équation 3,

$$
\phi_{p}^{*}\left(\frac{T_{b}}{T_{p f r}}\right)=\frac{\phi_{p}\left(\frac{T_{b}}{T_{p f r}}\right)}{\phi_{p}\left(\frac{T_{b}}{T_{p f r}}=0,63\right)}
$$

avec $\phi_{\mathrm{ch}}\left(\mathrm{T}_{\mathrm{b}} / \mathrm{T}_{\mathrm{pfr}}=0,63\right)=265 \mathrm{~kW} / \mathrm{m}^{2}$ et $\phi_{\text {fr }}\left(\mathrm{T}_{\mathrm{b}} / \mathrm{T}_{\mathrm{pfr}}=0,63\right)=169 \mathrm{~kW} / \mathrm{m}^{2}$. Le flux de référence est calculé pour un ratio de température de 0,63 , correspondant au flux le plus fort.

\subsubsection{Influence des incertitudes de mesures des températures de paroi sur les flux en fonction du ratio de température fluide-paroi}

L'effet de l'incertitude de mesure de la température de paroi chaude sur l'estimation des flux chaud et froid est observé sur la figure 4. Il apparaît que l'incertitude sur le flux augmente de façon croissante à mesure que le ratio des températures de fluide et de paroi froide tend vers 1. L'erreur relative commise, pour des ratios de températures proches de 1, est donc plus importante mais elle est associée à un flux plus faible. Malgré le fait qu'elle s'applique sur un flux plus faible, l'erreur absolue croît à mesure que la température de fluide se rapproche de la température de paroi froide. Son augmentation est, en revanche, quasi-linéaire.L'amélioration de la précision des mesures permet d'atténuer la pente des courbes pour un même ratio de température. La sous-estimation et la surestimation de la température de paroi chaude conduisent à des incertitudes semblables. Les incertitudes se propagent donc de façon symétrique par rapport à l'axe correspondant à une incertitude nulle. Les incertitudes sur la température de paroi chaude se propagent moins sur le flux de la paroi froide que sur le flux de la paroi chaude, comme observé sur la figure 2. Pour un ratio de température de 0,78 , une incertitude de $4 \%$ sur la température de paroi chaude conduit, tout de même, à une erreur légèrement supérieure à $3 \%$ sur le flux de la paroi froide.

L'influence de l'incertitude de mesure de la température de paroi froide sur l'estimation des flux chaud et froid est étudiée sur la figure 5. Les incertitudes sur le flux sont tracées en fonction du ratio $\mathrm{T}_{\mathrm{b}} /\left(\min \left(\mathrm{T}_{\mathrm{pfr}} ; \mathrm{T}_{\text {pfrexp }}\right)\right)$. En effet, lorsque la température de paroi froide mesurée, $\mathrm{T}_{\text {pfrexp }}$, est sousestimée, le ratio de température limitant pour la validité de la corrélation est le suivant $: T_{b} / T_{\text {pfrexp }}$. Si la température de paroi froide est sur-estimée, le ratio limitant est alors $T_{b} / T_{p f r}$. Comme observé sur la figure 4 , les incertitudes relatives augmentent de façon croissante à mesure que le ratio des températures de fluide et de paroi froide tend vers 1 . Les incertitudes absolues, non présentées dans cet article, croissent avec l'augmentation de la température de fluide. L'amélioration de la précision des mesures permet d'atténuer la pente des courbes pour un même ratio de température. Cependant, la dépendance de l'erreur sur les flux au signe de l'incertitude de mesure est plus marquée. Par exemple, à la paroi froide, pour un ratio de température de 0,73 , une sous-estimation, respectivement surestimation, de $8 \%$ de la température de paroi froide conduit à une incertitude de $14 \%$, respectivement $16,3 \%$, sur le flux. Côté 
chaud, l'erreur sur le flux reste inférieure à $4 \%$. Côté froid, les incertitudes de mesures s'amplifient et l'erreur sur le flux devient grande pour des incertitudes de mesures supérieures à $4 \%$.
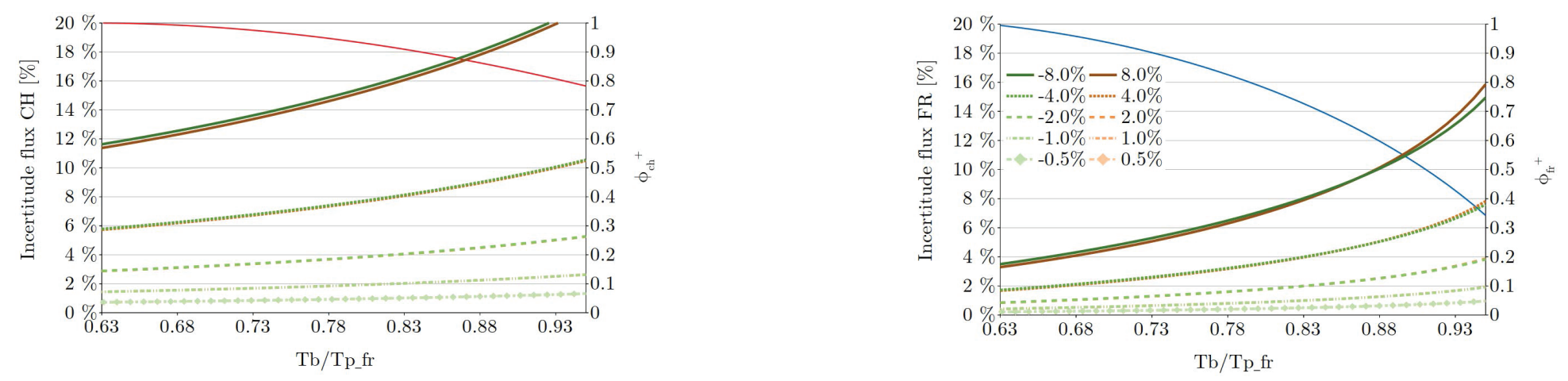

Figure 4. Influence de l'incertitude de mesure de la température de paroi chaude. Le graphique de gauche, respectivement droite, montre l'estimation du flux chaud, respectivement froid. La ligne rouge, repectivement bleu, décrit l'évolution du flux à la paroi chaude, respectivement froide, et est donc associée à l'axe secondaire du graphique.
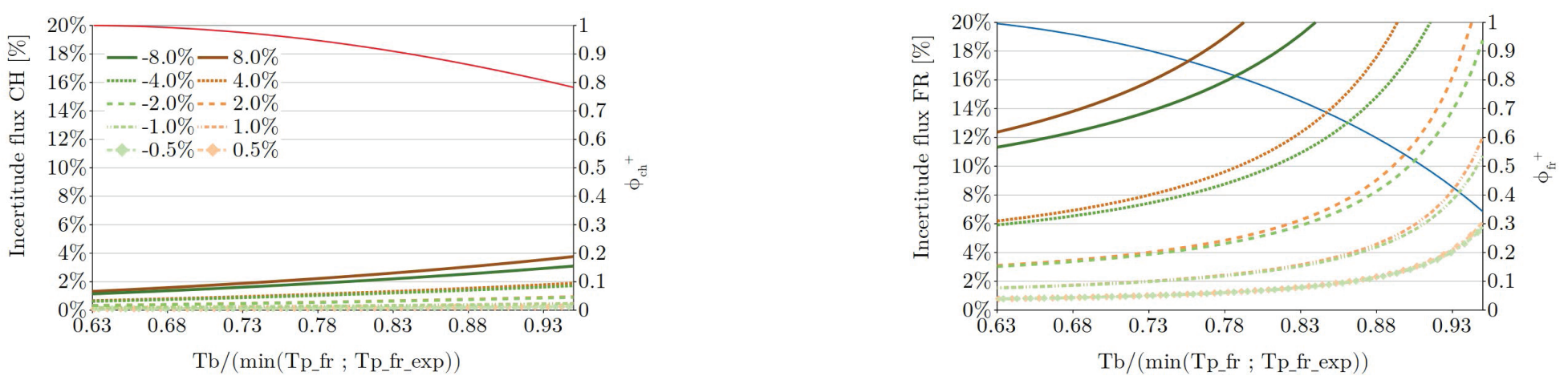

Figure 5. Influence de l'incertitude de mesure de la température de paroi froide. Le graphique de gauche, respectivement droite, montre l'estimation du flux chaud, respectivement froid. La ligne rouge, repectivement bleu, décrit l'évolution du flux à la paroi chaude, respectivement froide, et est donc associée à l'axe secondaire du graphique.

\subsubsection{Influence des incertitudes de mesures de la température de fluide sur les flux en fonction du ratio de température fluide-paroi}

L'effet de l'incertitude de mesure de la température de fluide est exposé sur la figure 6. En ce qui concerne l'influence de la précision des mesures, les mêmes comportements que ceux exposés sur les figures 4 et 5 sont observés. Côté froid, l'incertitude augmente avec le ratio de température $T_{b} / T_{\text {prr }}$ de façon croissante. Côté chaud, des tendances similaires sont observées mais dans une moindre mesure. L'évolution de l'incertitude sur le flux est très sensible au type d'erreur commise sur la température de fluide : la sous-estimation de la température de fluide conduit à de plus grands écarts que sa surestimation. Par exemple, côté chaud, pour un ratio de température de 0,88 , avec une incertitude de $8 \%$, en sousestimant la température de fluide on commet une erreur près de deux fois plus importante qu'en la surestimant $(11,8 \%$ contre $6,8 \%)$. 

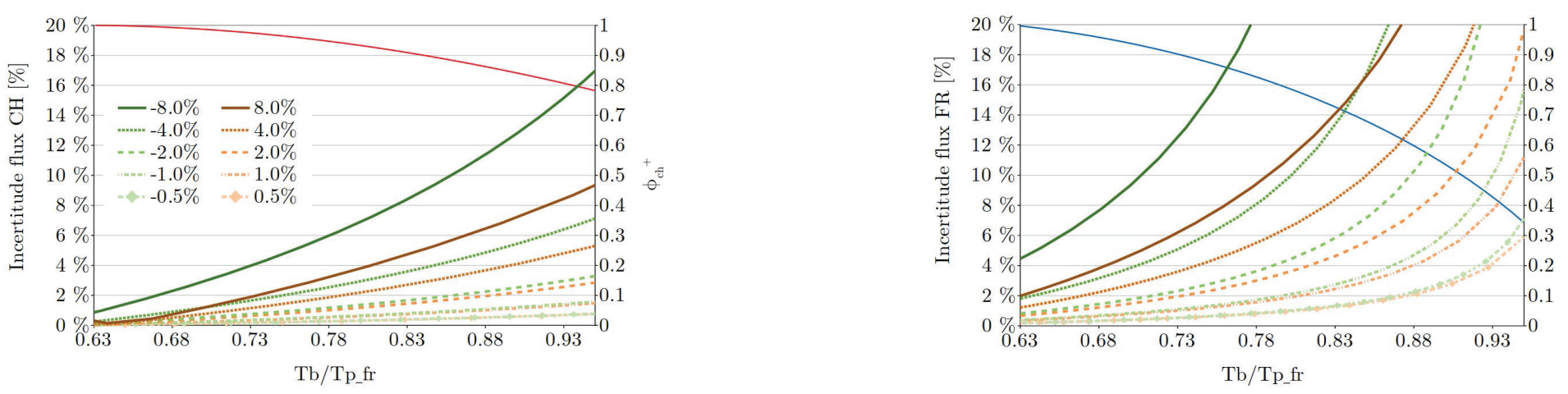

Figure 6. Influence de l'incertitude de mesure de la température de fluide. Le graphique de gauche, respectivement droite, montre l'estimation du flux chaud, respectivement froid. La ligne rouge, repectivement bleu, décrit l'évolution du flux à la paroi chaude, respectivement froide, et est donc associée à l'axe secondaire du graphique.

En ce qui concerne les incertitudes sur les nombres de Reynolds et de Prandtl, l'erreur sur les flux pariétaux est indépendante du ratio de température fluide-paroi.

\section{Conclusion}

Lors de ces travaux la corrélation de David et al. a été mise en œuvre. Elle permet d'estimer les transferts thermiques, dans des conditions de forts chauffages asymétriques d'un écoulement turbulent en canal caractérisé par de hauts niveaux de températures, avec une précision de $10 \%$. Puis une étude de sensibilité du flux aux paramètres de l'écoulement a été menée dans les conditions de fonctionnement des récepteurs solaires à gaz sous-pression des centrales solaires à tour. Enfin, l'influence du ratio des températures de fluide et de paroi a été analysée.

Les résultats obtenus montrent que les températures de paroi et de fluide sont très influentes sur l'estimation des flux pariétaux associés à ces parois. Les plus grandes incertitudes relatives sont obtenues sur le flux de la paroi froide pour des incertitudes sur les mesures de températures de paroi associée et de fluide. Le côté chaud est moins sensible à ces variations. Les incertitudes de mesures sur la paroi opposée induisent des erreurs sur l'estimation du flux. La pente de la propagation des erreurs sur les flux a été estimée pour différents niveau de température de paroi, représentant différentes positions axiales dans le récepteur solaire. Il apparaît que l'erreur croît avec l'augmentation des niveaux de température lorsque les écarts entre les températures sont constantes. Les incertitudes relatives sur les flux augmentent de façon croissante à mesure que le ratio de température fluide-paroi froide tend vers 1 . Néanmoins, étant donné que le flux suit une tendance opposée, les plus grandes erreurs sont commises pour les flux les plus faibles.

\section{Bibliographie}

[1] E. Battista et H. C. Perkins, Turbulent heat and momentum transfer in a square duct with moderate property variations, International Journal of Heat and Mass Transfer, vol. 13, no. 6, p. 1063-1065, 1970.

[2] A. P. Colburn, A method of correlating forced convection heat-transfer data and a comparison with fluid friction, International Journal of Heat and Mass Transfer, vol. 7, no. 12, p. 1359-1384, 1964.

[3] F. W. Dittus et L. M. K. Boelter, Heat transfer in automobile radiators of the tubular type, International Communications in Heat and Mass Transfer, vol. 12, no. 1, p. 3-22, 1985. 
[4] V. Gnielinski, New equations for heat and mass transfer in turbulent pipe and channel flow, International Journal of Chemical Engineering, vol. 16, no. 2, p. 359-368, 1976.

[5] D. Jo, O. S. Al-Yahia, R. M. Altamimi, P. Jong-Hark et C. H. Taek, Experimental investigation of convective heat transfer in a narrow rectangular channel for upward and downward flows, Nuclear Engineering and Technology, vol. 46, no. 2, p. 195-206, 2014.

[6] X. Daguenet-Frick, A. Toutant, F. Bataille et G. Olalde, Numerical investigation of a ceramic high-temperature pressurized-air solar receiver, Solar Energy, vol. 90, p. 164-178, 2013.

[7] A. Håkansson, An investigation of uncertainties in determining convective heat transfer during immersion frying using the general uncertainty management framework, Journal of Food Engineering, vol. 263, p. 424-436, 2019.

[8] JCGM (Joint Committee for Guides in Metrology), Evaluation of measurement data - Guide to the expression of uncertainty in measurement, JCOM100:2008.

[9] E. Driscoll et D. Landrum, Uncertainty Analysis on Heat Transfer Correlations for RP-1 Fuel in Copper Tubing, rap. tech., 2004.

[10] V. K. Scariot, G. M. Hobold et A. K. da Silva, On the sensitivity to convective heat transfer correlation uncertainties in supercritical fluids, Applied Thermal Engineering, vol. 145, p. 123-132, 2018.

[11] M. David, A. Toutant et F. Bataille, Numerical development of heat transfer correlation in asymmetrically heated turbulent channel flow, International Journal of Heat and Mass Transfer, vol. 164, p. 120599, 2021.

[12] W. Rozema, H. J. Bae, P. Moin et R. Verstappen, Minimum-dissipation models for large-eddy simulation, Physics of Fluids, vol. 27, no. 8, p. 085107, 2015.

[13] J. Capeillère, A. Toutant, G. Olalde et A. Boubault, Thermomechanical behavior of a plate ceramic solar receiver irradiated by concentrated sunlight, Solar Energy, vol. 110, p. 174-187, 2014.

\section{Remerciements}

Ces travaux ont bénéficié d'un accès aux moyens de calcul du CINES au travers de l'allocation de ressources A0082A05099 attribuée par GENCI (Grand Équipement National de Calcul Intensif). Nous remercions le CEA pour la mise à disposition de la plateforme TRUST et le développement du code de calcul TrioCFD (open source). Nous remercions également la région Occitanie pour sa participation au financement de ces travaux de thèse. 